\title{
UTILIZAÇÃO DE PSICOFÁRMACOS E SINTOMAS DE ANSIEDADE E DEPRESSÃO EM ESTUDANTES UNIVERSITÁRIOS DE LAGARTO/SE DURANTE A PANDEMIA DA COVID-19
}

Wilma Caitano dos Santos ${ }^{1 *}$

Edimes Mikaele Sá Dantas dos Santos²

Karenine Maria Holanda Cavalcante ${ }^{3}$

\author{
${ }^{1}$ Farmacêutica pela Universidade Federal de Sergipe (UFS). Lagarto, Sergipe, Brasil \\ wilmacaetano17@gmail.com \\ https://orcid.org/0000-0002-3481-6273 \\ ${ }^{2}$ Acadêmica de enfermagem pela Universidade Federal de Sergipe (UFS). Lagarto, Sergipe, Brasil. \\ edimes.jesus@gmail.com \\ https://orcid.org/0000-0001-5263-2411 \\ ${ }^{3}$ Doutora em Ciências Fisiológicas pela Universidade Federal de Sergipe, Professora Adjunta do curso de \\ enfermagem da Universidade Federal de Sergipe (UFS). Lagarto, Sergipe, Brasil. \\ karenine@academico.ufs.br \\ https://orcid.org/0000-0003-0840-6833
}

\section{RESUMO}

Introdução: A pandemia causada pela COVID-19 pode provocar impactos na saúde mental dos universitários, devido a fatores como suspensão das aulas presenciais e a sobrecargas do ensino online, assim objetivou-se quantificar o número de estudantes universitários com ansiedade e depressão provável relacionando com o uso de psicofármacos durante a pandemia. Métodos: Estudo transversal, descritivo e quantitativo aprovado pelo CEP (CAAE: 37453920.0.0000.5546). Foi aplicado um questionário online para verificação dos sintomas de depressão (Questionário Sobre Saúde do Paciente; PHQ-2) e ansiedade (Escala de Transtorno geral de Ansiedade; GAD-2), e sobre o uso de psicofármacos e perfil sociodemográfico de estudantes de graduação da Universidade Federal de Sergipe (UFS) de Lagarto-SE. Resultados: Dos 99 participantes, 48,5\% foram classificados com ansiedade provável (GAD-2 $\geq 3$ ) e 37,4\% com depressão provável (PHQ-2 $\geq 3$ ), enquanto 33,3\% ( $n=33)$ apresentaram depressão e ansiedade provável, simultaneamente. Destes 10,1\% $(n=10)$ faziam uso de psicofármacos. Uma quantidade significativa de pessoas que não fazem uso de psicofármacos apresentou sintomas de ansiedade $(47,2 \%, n=42)$ e de depressão $(33,7 \%, n=30)$. Conclusão: A grande maioria dos estudantes foi avaliada com depressão ou ansiedade provável, entretanto, uma pequena porcentagem faz uso de psicofármacos, podendo sugerir outras formas de tratamentos ou a falta de tratamento. Assim é sugerido que os mesmos procurem por apoio psicológico para avaliações adicionais.

Palavras-chave: ansiedade; COVID-19; depressão; estudantes; psicofármacos.

*Autor correspondente: Wilma Caitano dos Santos, Farmacêutica pela Universidade Federal de Sergipe, Lagarto, Sergipe, Brasil. (79) 99812-8278; caetanowilma22@gmail.com.

https://doi.org/10.51161/rems/2391

Editora IME@ 2021. Todos os direitos reservados. 


\section{INTRODUÇÃO}

Segundo a Organização Mundial da Saúde (OMS) (2020) a doença Coronavirus Disease-2019 (COVID-19) provocada pelo vírus SARS-CoV-2 (causador da síndrome respiratória aguda grave-2) foi identificada pela primeira vez em dezembro de 2019 na cidade de Wuhan, na China, e em janeiro de 2020 a OMS declarou-a como uma emergência em saúde pública de interesse internacional, que é - mais alto nível de alerta da organização (MAIA; DIAS, 2020; OPAS/OMS, 2020).

$\mathrm{O}$ primeiro caso dessa doença no Brasil foi registrado no dia 26 de fevereiro de 2020, e em 11 de março de 2020, a COVID-19 foi caracterizada pela OMS como uma pandemia (BRASIL, 2020; MAIA; DIAS, 2020). Após 3 dias (dia 14 março de 2020) foi registrado o primeiro caso em Sergipe, e até o momento (12 agosto de 2021) foram registrados 276.653 casos e 5.946 óbitos por COVID-19 neste estado (SES, 2020, 2021).

Mesmo nos meses iniciais de pandemia a OMS já alertava para o fato desta crise estar gerando estresse na população mundial (MAIA; DIAS, 2020). Estudos que avaliaram as implicações do COVID-19 na saúde mental identificaram uma prevalência elevada de sintomatologia depressiva e ansiosa autorrelatada de moderada a grave na população em geral, refletindo os efeitos generalizados de incerteza e medos relacionados com a pandemia (GRUBIC; BADOVINAC; JOHRI, 2020).

Segundo a OMS (2018) a saúde mental é um estado de bem-estar no qual um indivíduo realiza suas próprias habilidades, pode lidar com o estresse normal da vida, trabalhar produtivamente e ser capaz de contribuir com sua comunidade (PEREIRA et al., 2020). Esta pode ser afetada pelo distanciamento social, que consiste em estratégias que evitam a propagação do vírus causador da doença, tais como: evitar aglomerações, manter um distanciamento de no mínimo um metro e meio entre as pessoas, e a proibição de eventos e locais com um grande número de indivíduos reunidos (por exemplo: escolas, universidades, shoppings e shows, entre outros) (PEREIRA et al., 2020).

Como resultado dessas medidas de distanciamento social implementadas em resposta ao COVID-19, as instituições de ensino superior suspenderam as aulas presenciais e mudaram para um formato de aprendizagem online (GRUBIC; BADOVINAC; JOHRI, 2020; MAIA; DIAS, 2020). Com essas mudanças provocadas no formato das aulas ou suspensão das mesmas, existe a possibilidade dos estudantes universitários experimentarem a diminuição da motivação para os estudos, dificuldade de adaptação, aumento das pressões para aprender, abandono das rotinas diárias e taxas mais altas de evasão, e consequentemente causar $\mathrm{o}$ aumento de estressores acadêmicos e dos níveis de ansiedade e depressão (GRUBIC; BADOVINAC; JOHRI, 2020; MAIA; DIAS, 2020).

Segundo a American College Health Association (2006) boa parte dos estudantes universitários apresentam depressão $(17,5 \%)$ e transtorno de ansiedade $(12,7 \%)$ corriqueiramente; e embora haja necessidade de prescrição de medicamentos para o tratamento desses transtornos, muitos destes estudantes utilizam esses medicamentos de forma indevida e abusiva (STONE; MERLO, 2011).

Os medicamentos comumente prescritos para o tratamento de transtornos mentais, tais como: estimulantes, benzodiazepínicos e antidepressivos são utilizados pelos estudantes de forma não médica para o aumento de desempenho, alívio de estresse e produção de euforia (STONE; MERLO, 2012).

Resultados do estudo realizado por Maia et al., (2020) confirmaram um aumento significativo de perturbação psicológica (ansiedade, depressão e estresse) entre os estudantes universitários no período pandêmico comparativamente a períodos normais (MAIA; DIAS, 2020).

Diante do exposto, é possível inferir que estudantes universitários apresentam em seu cotidiano fatores estressores que podem afetar a sua saúde mental e aumentar o consumo de medicamentos psicofármacos, portanto, considerando os impactos do período pandêmico na rotina acadêmica, o presente estudo visou quantificar o número de 
estudantes universitários com sintomas de depressão e ansiedade relacionado com o uso de psicofármacos durante a pandemia da COVID-19.

\section{MATERIAL E MÉTODO}

Trata-se de um estudo transversal de abordagem descritiva e quantitativa realizado com estudantes da Universidade Federal de Sergipe (UFS), Campus Universitário Professor Antônio Garcia Filho, localizado em Lagarto Sergipe, que é composto por cursos da área da saúde. Este estudo foi aprovado pelo Comitê de Ética em Pesquisa envolvendo Seres Humanos da Universidade Federal de Sergipe (CEP-UFS), sob o parecer $\mathrm{n}^{\circ} 4.449 .710$ e CAAE n०37453920.0.0000.5546.

Foram incluídos na pesquisa discentes maiores de 18 anos, de todos os cursos a partir do $2^{\circ}$ ano/ciclo da graduação e que aceitaram participar da pesquisa através da assinatura do Termo de Consentimento Livre e Esclarecido (TCLE). Foram excluídos da pesquisa questionários incompletos (com uma ou mais questões sem resposta sobre a utilização de medicamentos, transtornos de ansiedade e depressão provável), e discentes de Pósgraduação, Mestrado e Doutorado.

A coleta dos dados ocorreu durante os meses de dezembro de 2020 a fevereiro de 2021, foi realizada por meio da aplicação de 4 questionários online: o primeiro questionário adaptado de Souza (2017) serviu para conhecer o perfil sociodemográfico da amostra (sexo biológico, idade, curso, renda individual e escolaridade), o segundo foi adaptado de Schneider e Azambuja (2015) serviu para descrever a utilização de psicofármacos (nome do psicofármaco utilizado, tempo e motivo do uso) (SCHNEIDER; AZAMBUJA, 2015; SOUZA, 2017).

Enquanto que o terceiro tratou-se da escala de Transtorno geral de Ansiedade (GAD-2), proposta por Kroenke et al., (2007), que avaliou a presença de ansiedade provável, este apresenta pontuação total que varia entre 0 a 6, aqueles entrevistados que tiverem pontuação no total maior ou igual 3 foram classificados com provável ansiedade
(KROENKE et al., 2007; NI et al., 2020).

No que se refere ao quarto, tratou-se do Questionário Sobre Saúde do Paciente (PHQ2), proposto por Kroenke et al., (2003), que avaliou a presença de depressão provável; neste a pontuação total varia de 0 a 6 , aqueles entrevistados que tiveram pontuação maior ou igual a 3 foram classificados com provável depressão (KROENKE; SPITZER; WILLIAMS, 2003; NI et al., 2020).

Os termos ansiedade prováveis e depressão provável foram utilizados, devido ao fato dos questionários GAD-2 e PHQ-2 serem breves instrumentos de triagem validados, que não podem ser utilizados para o diagnóstico de transtornos mentais (NI et al., 2020).

Os dados foram computados em uma planilha do Excel e analisados no programa Open Source Epidemiologic Statistics for Public Health, Versão 3.01 gratuita, sendo calculadas a frequência, absoluta e relativa, e as médias das variáveis e estas apresentadas em gráficos e tabelas. Para avaliar a significância estatística da associação entre as variáveis, foi utilizado o teste do Qui quadrado com um intervalo de confiança de $95 \%$.

Esta pesquisa atendeu aos fundamentos éticos e científicos pertinentes a resolução $N^{\circ}$ 510 (7 de Abril de 2016) e a resolução 466 (12 de dezembro de 2012) no que tange respeito ao participante da pesquisa em sua dignidade $\mathrm{e}$ autonomia, informando e reforçando seus direitos, reconhecendo sua vulnerabilidade, assegurando sua vontade de contribuir e permanecer, ou não, na ação, por intermédio de manifestação expressa, livre e esclarecida (BRASIL, 2016).

\section{RESULTADOS}

A partir das respostas dos 99 participantes, a idade média dos participantes foi de 22,3 anos (+/- 2,6 anos), sendo a maioria: do sexo feminino $(71,7 \%, n=71)$, do $2^{\circ}$ ano $(31,3 \%, n=31)$, do curso de enfermagem $(22,2 \%, n=22)$, solteiro $(94,95, n=94 \%)$, recebem auxílio financeiro dos pais $(81,82 \%, n=81)$, pardo $(62,6 \%, n=62)$, sem renda mensal individual $(43,4 \%, n=43)$, e residiam com a família $(77,78 \%, \mathrm{n}=77$ ) (tabela 1).

A análise dos achados sociodemográficos 
revelou uma maior prevalência de sintomas de ansiedade em mulheres, adultos de 22 a 36 anos, do $2^{\circ}$ ano, do curso de enfermagem, solteiros, pardos, com auxílio financeiro dos pais, com nenhuma renda mensal individual $e$ que moram com família (tabela 2).

Os sintomas de depressão foram mais prevalentes entre mulheres, jovens de 18 a 22 anos, do $2^{\circ}$ ano, do curso de terapia ocupacional, solteiros, pardos, com auxílio financeiro dos pais, com nenhuma renda mensal individual, e que moram com família (tabela 2). Além disso, foi observado apenas associação estatística entre depressão e 0 ano de estudo (Qui-quadrado $=9,458, p$-valor $=0,02378$ ).

Tabela 1 - Distribuição das características sociodemográficas dos universitários ( $n=99$ ). Lagarto, Sergipe, Brasil, dezembro de 2020 a fevereiro de 2021.

\begin{tabular}{|c|c|c|c|}
\hline Variáveis & Categorias & $N$ & $\%$ \\
\hline \multirow[t]{2}{*}{ Sexo } & Feminino & 71 & 71,7 \\
\hline & Masculino & 28 & 28,3 \\
\hline \multirow[t]{2}{*}{ Idade (anos) } & $18-21$ & 42 & 42,4 \\
\hline & $22-36$ & 57 & 57,6 \\
\hline \multirow[t]{4}{*}{ Ano } & $2^{\circ}$ & 31 & 31,3 \\
\hline & $3^{\circ}$ & 22 & 22,2 \\
\hline & $4^{\circ}$ & 23 & 23,2 \\
\hline & $5^{\circ}$ & 23 & 23,2 \\
\hline \multirow[t]{8}{*}{ Curso } & Enfermagem & 22 & 22,2 \\
\hline & Terapia Ocupacional & 18 & 18,2 \\
\hline & Farmácia & 18 & 18,2 \\
\hline & Medicina & 15 & 15,2 \\
\hline & Fisioterapia & 10 & 10,1 \\
\hline & Fonoaudiologia & 8 & 8,1 \\
\hline & Nutrição & 5 & 5,1 \\
\hline & Odontologia & 3 & 3,0 \\
\hline \multirow[t]{2}{*}{ Estado civil } & Solteiro (a) & 94 & 95,0 \\
\hline & Casado (a) & 5 & 5,0 \\
\hline \multirow[t]{3}{*}{ Etnia/raça } & Pardo & 62 & 62,6 \\
\hline & Branco & 28 & 28,3 \\
\hline & Preto & 9 & 9,1 \\
\hline \multirow[t]{4}{*}{ Auxílio financeiro } & Pais ajudam financeiramente & 81 & 81,8 \\
\hline & Auxílio/bolsa da UFS & 8 & 8,1 \\
\hline & Emprego autônomo & 8 & 8,1 \\
\hline & Outros & 2 & 2,0 \\
\hline \multirow{5}{*}{$\begin{array}{l}\text { Renda mensal individual } \\
\text { (salário-mínimo*) }\end{array}$} & Nenhuma & 43 & 43,4 \\
\hline & $<1$ & 40 & 40,4 \\
\hline & $>1 \mathrm{a}<1=3$ & 13 & 13,1 \\
\hline & $>3 a</=5$ & 2 & 2,0 \\
\hline & $>5$ & 1 & 1,0 \\
\hline \multirow[t]{4}{*}{ Residência } & Família nuclear (pai, mãe, irmãos) & 77 & 77,8 \\
\hline & Amigos/colegas & 10 & 10,1 \\
\hline & Sozinho & 7 & 7,1 \\
\hline & Outros & 5 & 5,1 \\
\hline
\end{tabular}

*Salário-mínimo: 1,045,00 vigente em 2020. Fonte: Autoria Própria. 
Tabela 2- Distribuição da prevalência dos estudantes com ansiedade e depressão de acordo com as características sociodemográficas (n=99). Lagarto, Sergipe, Brasil, dezembro de 2020 a fevereiro de 2021.

\begin{tabular}{|c|c|c|c|c|c|}
\hline \multirow{2}{*}{ Variáveis } & \multirow{2}{*}{ Categorias } & \multicolumn{2}{|c|}{$\begin{array}{l}\text { Ansiedade pro- } \\
\text { vável }\end{array}$} & \multicolumn{2}{|c|}{$\begin{array}{l}\text { Depressão pro- } \\
\text { vável }\end{array}$} \\
\hline & & $\operatorname{Sim}_{N}$ & $\%$ & $\underset{N}{\operatorname{Sim}}$ & $\%$ \\
\hline \multirow[t]{2}{*}{ Sexo } & Feminino & 35 & 72,9 & 25 & 67,6 \\
\hline & Masculino & 13 & 27,1 & 12 & 32,4 \\
\hline \multirow[t]{2}{*}{ Idade (anos) } & $18-21$ & 22 & 45,8 & 19 & 51,4 \\
\hline & $22-36$ & 26 & 56,2 & 18 & 48,6 \\
\hline \multirow[t]{4}{*}{ Ano } & $2^{\circ}$ & 17 & 35,4 & 17 & 45,9 \\
\hline & $3^{\circ}$ & 14 & 29,2 & 10 & 27,1 \\
\hline & $4^{\circ}$ & 8 & 16,7 & 5 & 13,5 \\
\hline & $5^{\circ}$ & 9 & 18,7 & 5 & 13,5 \\
\hline \multirow[t]{8}{*}{ Curso } & Enfermagem & 12 & 25,0 & 6 & 16,2 \\
\hline & Terapia Ocupacional & 9 & 18,7 & 7 & 18,9 \\
\hline & Farmácia & 8 & 16,7 & 6 & 16,2 \\
\hline & Medicina & 8 & 16,7 & 6 & 16,2 \\
\hline & Fisioterapia & 4 & 8,3 & 4 & 10,8 \\
\hline & Fonoaudiologia & 5 & 10,4 & 5 & 13,5 \\
\hline & Nutrição & 1 & 2,1 & 2 & 5,4 \\
\hline & Odontologia & 1 & 2,1 & 1 & 2,8 \\
\hline \multirow[t]{2}{*}{ Estado civil } & Solteiro (a) & 46 & 95,8 & 37 & 100 \\
\hline & Casado (a) & 3 & 4,2 & 0 & 0 \\
\hline \multirow[t]{3}{*}{ Etnia/raça } & Pardo & 30 & 62,5 & 24 & 64,9 \\
\hline & Branco & 14 & 29,2 & 10 & 27,0 \\
\hline & Preto & 4 & 8,3 & 3 & 8,1 \\
\hline \multirow[t]{4}{*}{ Auxílio financeiro } & Pais ajudam financeiramente & 41 & 85,4 & 32 & 84,5 \\
\hline & Auxílio/bolsa da UFS & 4 & 8,3 & 2 & 5,4 \\
\hline & Emprego autônomo & 3 & 6,3 & 3 & 8,2 \\
\hline & Outros & 0 & 0,0 & 0 & 0,0 \\
\hline \multirow{5}{*}{$\begin{array}{l}\text { Renda mensal indivi- } \\
\text { dual (salário-mínimo*) }\end{array}$} & Nenhuma & 22 & 45,8 & 20 & 54,1 \\
\hline & $<1$ & 17 & 35,4 & 9 & 24,3 \\
\hline & $>1 \mathrm{a}<1=3$ & 8 & 16,6 & 7 & 18,9 \\
\hline & $>3 \mathrm{a}</=5$ & 1 & 2,0 & 1 & \\
\hline & $>5$ & 0 & 0 & 0 & 0,0 \\
\hline \multirow[t]{4}{*}{ Residência } & $\begin{array}{l}\text { Família nuclear (pai, mãe, ir- } \\
\text { mãos) }\end{array}$ & 39 & 81,3 & 31 & 83,8 \\
\hline & Amigos/colegas & 4 & 8,3 & 2 & 5,4 \\
\hline & Sozinho & 3 & 6,2 & 3 & 8.1 \\
\hline & Outros & 2 & 4,2 & 1 & 2,7 \\
\hline
\end{tabular}

*Salário-mínimo: 1,045,00 vigente em 2020. Fonte: Autoria Própria. 
Dos 99 universitários, $48 \% \quad(n=48)$ foram classificados com ansiedade provável e $37 \%$. $(n=37)$ com depressão provável (Gráfico 1). Dos que fizeram uso de psicofármacos ( $n=$ 10), $60,0 \%(n=6)$ apresentaram ansiedade provável e $70,0 \% \quad(n=7)$ depressão provável. Uma quantidade significativa de pessoas que não fazem uso de psicofármacos $(n=89)$ apresentou ansiedade provável $(47,2 \%, n=42)$ e $33,7 \%(n=30)$ depressão provável. Enquanto que $33,3 \%(n=33)$ apresentaram depressão e ansiedade provável, concomitantemente, destes $18,2 \%(n=6)$ participantes faziam uso de psicofármacos e $81,8 \%(n=27)$ não faziam uso.

Figura 1 - Distribuição da classificação dos universitários quanto à presença de sintomas de provável ansiedade e depressão (n=99). Lagarto, Sergipe, Brasil, dezembro de 2020 a fevereiro de 2021

\section{Ansiedade Provável e Depressão Provável}

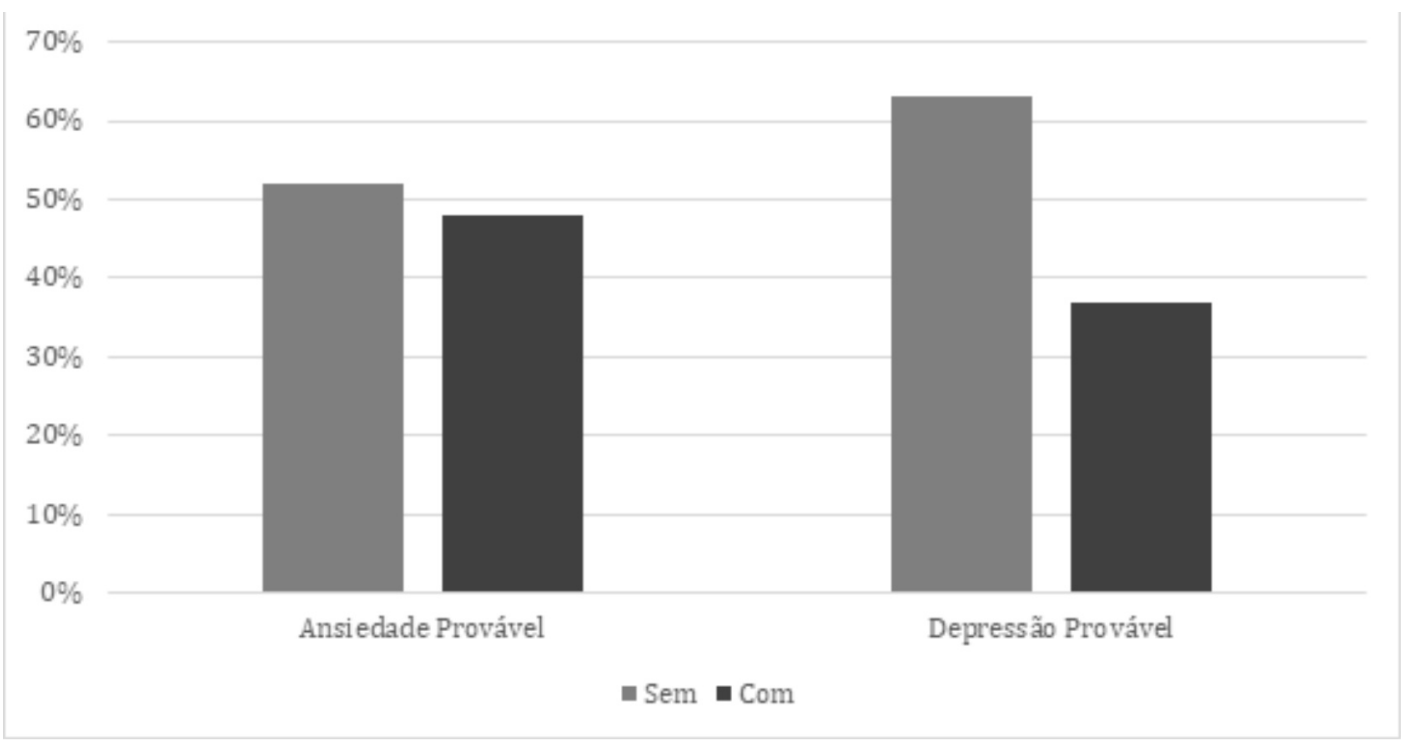

Fonte: Autoria Própria.

$\mathrm{Na}$ categoria da escala (quase todos os dias $), \quad 28,3 \% \quad(n=28)$ sentiram-se nervoso, ansioso ou muito tenso; $20,2 \% \quad(n=20)$, consideraram-se incapaz de parar/controlar as preocupações; $17,2 \%(n=17)$ relataram pouco interesse/prazer em fazer as coisas e $16,2 \%$ $(n=16)$ afirmaram sentir-se "pra baixo", deprimido(a)/sem perspectiva (tabela 3).

Entre os participantes da pesquisa, $10,10 \%(n=10)$ faziam uso de psicofármacos, sendo identificados 15 psicofármacos. A classe mais utilizada foi a dos antidepressivos $(60,00 \%, n=9)$, e a segunda a dos ansiolíticos
$(20,00 \%, \mathrm{n}=3)$ (tabela 4).

Do total de 10 participantes que utilizam psicofármacos $(10,1 \%)$, a maioria relatou apresentar três ou mais transtornos mentais associados (50\%, $\mathrm{n}=5)$, enquanto, $30,00 \%$ $(n=3)$ relataram apresentar ansiedade. Houve uma maior prevalência de participantes que utilizam os psicofármacos de 6 a 11 meses, e o segundo maior tempo de utilização foi de 1 a 2 anos $(30 \%, n=3)$, além disso, houve um maior número de participantes que não associaram 0 uso de psicofármacos a pandemia da COVID-19 $(80 \%, \mathrm{n}=8)$ (tabela 5). 
Tabela 3- Distribuição dos sintomas de ansiedade e depressão dos universitários conforme escalas GAD-2 e PHQ-2. Lagarto, Sergipe, Brasil, dezembro de 2020 a fevereiro de 2021.

\begin{tabular}{cccc}
\hline Variáveis (Escala GAD-2) & Categoria & $\mathrm{N}$ & $\%$ \\
\hline Sentindo-se nervoso, ansioso ou muito tenso & Nenhuma vez & 13 & 13,1 \\
& Vários dias & 40 & 40,4 \\
Não ser capaz de parar ou controlar as preocupações & Mais da metade dos dias & 18 & 18,2 \\
& Quase todos os dias & 28 & 28,3 \\
& Nenhuma vez & 27 & 27,3 \\
Variáveis (Escala PHQ-2) & Vários dias & 34 & 34,3 \\
Pouco interesse ou prazer em fazer as coisas & Mais da metade dos dias & 18 & 18,2 \\
& Quase todos os dias & 20 & 20,2 \\
& & & \\
Se sentir 'pra baixo', deprimido/a ou sem perspectiva & Categoria & $\mathrm{N}$ & $\%$ \\
& Nenhuma vez & 27 & 27,3 \\
& Vários dias & 41 & 41,4 \\
& Mais da metade dos dias & 14 & 14,1 \\
& Quase todos os dias & 17 & 17,2 \\
& Nenhuma vez & 28 & 28,3 \\
& Vários dias & 36 & 36,4 \\
& Mais da metade dos dias & 19 & 19,2 \\
& Quase todos os dias & 16 & 16,2 \\
\hline
\end{tabular}

Fonte: Autoria Própria.

Tabela 4- Distribuição dos medicamentos utilizados pelos universitários $(n=15)$. Lagarto, Sergipe, Brasil, dezembro de 2020 a fevereiro de 2021.

\begin{tabular}{c|c|c}
\hline Classificação ATC dos psicofármacos & N & $\%$ \\
\hline Antidepressivo (N06A) & 9 & 60,0 \\
Sertralina & 2 & 13,3 \\
Trazodona & 2 & 13,3 \\
Fluoxetina & 2 & 13,3 \\
Fluvoxamina & 1 & 6,7 \\
Bupropiona & 1 & 6,7 \\
Escitalopram & 1 & 6,7 \\
Ansiolítico (N05B) & 3 & 20,0 \\
Clonazepam & 2 & 13,3 \\
Alprazolam & 1 & 6,7 \\
Antipsicótico (N05A) & 1 & 6,7 \\
Quetiapina & 1 & 6,7 \\
Estimulantes psicomotores (N06B) & 1 & 6,7 \\
Metilfenidato & 1 & 6,7 \\
Antiepilético (N03AX) & 1 & 6,7 \\
Topiramato & 1 & 6,7 \\
\hline
\end{tabular}

Fonte: Autoria Própria. 
Tabela 5 - Distribuição do motivo e tempo de uso dos psicofármacos pelos universitários e a associação desse uso com a pandemia da COVID-19 ( $n=10)$. Lagarto, Sergipe, Brasil, dezembro de 2020 a fevereiro de 2021.

\begin{tabular}{|c|c|c|}
\hline Motivo de uso dos psicofármacos & $\mathrm{N}$ & $\%$ \\
\hline Transtornos associados & 5 & 50,0 \\
\hline Ansiedade, depressão, insônia e síndrome do pânico & 2 & 20,0 \\
\hline Ansiedade, depressão, dor intensa e insônia & 1 & 10,0 \\
\hline Ansiedade, depressão, insônia e TDAH* & 1 & 10,0 \\
\hline Ansiedade, depressão e insônia & 1 & 10,0 \\
\hline Ansiedade & 3 & 30,0 \\
\hline TDAH & 1 & 10,0 \\
\hline Transtorno bipolar & 1 & 10,0 \\
\hline Tempo de uso dos psicofármacos & $\mathrm{N}$ & $\%$ \\
\hline 6 a 11 meses & 4 & 40,0 \\
\hline 1 a 2 anos & 3 & 30,0 \\
\hline 3 a 4 anos & 1 & 10,0 \\
\hline$>5$ anos & 1 & 10,0 \\
\hline 1 a 5 meses & 1 & 10,0 \\
\hline Associam o uso com a pandemia da COVID-19 & $\mathrm{N}$ & $\%$ \\
\hline Não & 8 & 80,0 \\
\hline Sim & 2 & 20,0 \\
\hline
\end{tabular}

Fonte: Autoria Própria.

\section{DISCUSSÃO}

A presente pesquisa constatou maior prevalência de ansiedade e depressão entre as mulheres. Normalmente as pesquisas mostram maior prevalência de sintomas de ansiedade e depressão em mulheres e atribuem ao fato de que as mesmas são mais sensíveis ao estresse psicológico por fatores hormonais, como também, ao pouco tempo dedicado à diminuição do estresse, devido à sobrecarga com outras atividades como os afazeres domésticos (ALSHARJI, 2020; BASHIR et al., 2020).

No que se refere a idade, a maior prevalência de ansiedade e depressão nos mais jovens, pode-se propor a possibilidade de uma maior exposição desse segmento da população às notícias das redes sociais/mídia digital, que geralmente são sobrecarregadas com notícias falsas e informações não confirmadas, que pode influenciar o estresse bem como distúrbios hormonais relacionados à idade ou limitação de atividades ao ar livre (ALSHARJI, 2020; BASHIR et al., 2020; GUALANO et al., 2020; LIU et al., 2020;
MOUSTAFA et al., 2017).

Além disso, a suspensão das aulas presenciais nas universidades pode ter imposto sentimentos de solidão, tédio e incerteza (ALSHARJI, 2020; SANTINI et al., 2020). Esses sentimentos negativos são geralmente associados a implicações psicológicas adversas, incluindo ansiedade e depressão (ALSHARJI, 2020).

Foi observado associação estatística entre depressão e o ano de estudo. A maior frequência dos sintomas de ansiedade $e$ depressão no segundo ano, pode estar relacionado ao fato de que neste ano os alunos iniciam o conhecimento de maneira mais aprofundada e específica sobre a profissão escolhida, momento que pode provocar sentimentos de incertezas e inseguranças sobre a futura profissão e nas práticas do curso potencializados com ensino remoto (ROLLEMBERG, 2018). Além disso, há o fato de que os estudantes podem ter tido incertezas e receios quanto aos atrasos na colação de grau devido com a suspensão das aulas presenciais (ROLLEMBERG, 2018).

Cursos como Enfermagem, Terapia 
Ocupacional, Medicina e Fonoaudiologia apresentaram frequência relativa de ansiedade provável igual ou acima de $50 \%$. Não foram encontrados estudos que apresentassem resultados comparativos acerca de transtornos mentais entre esses cursos da saúde. Entretanto, é importante destacar que devido o estigma imposto pela sociedade, os estudantes universitários, apresentam dificuldades em recorrer tratamentos de transtornos mentais, por insegurança ou medo, assim, resistem em desmontar fragilidade diante a sociedade, assim, aponta a necessidade de implementação de intervenções de saúde mental, haja vista, a importância desses futuros profissionais no cuidado aos pacientes (LELIS et al., 2020; MENDONÇA, 2020).

Houve uma maior frequência de pardos e solteiros com sintomas de ansiedade e depressão. Essa prevalência maior nos pardos pode estar associada a maior probabilidade de sofrer experiências de discriminação (SMOLEN; ARAÚJO, 2017). Outrossim, a frequência maior de solteiros também foi evidenciada em um estudo com universitários no Acre, podendo ser em decorrência da faixa etária da amostra, e devido a muitos não terem renda própria (SANTIAGO et al., 2021).

A maior prevalência em estudantes com renda baixa com sintomas de transtornos mentais, pode ter sido ao repentino aumento da insegurança financeira advindo da pandemia, que colocou os universitários em uma situação desagradável, afetando seu bemestar socioeconômico e mental (ISLAM et al., 2020; VAN HARMELEN et al., 2016).

Os resultados sugerem que apesar de viver com a família, os sintomas de ansiedade e depressão tem sido evidenciado entre os estudantes universitários, que pode ser principalmente devido à insegurança financeira (ISLAM et al., 2020). Morar com a família pode gerar forte tranquilidade entre os indivíduos, e consequentemente, reduzir esses sintomas (ISLAM et al., 2020; VAN HARMELEN et al., 2016). No entanto, esta pandemia trouxe extrema pressão e insegurança financeira às famílias que têm sofrido com a queda na renda e dívidas incontroláveis, assim os universitários que antes contribuíam com suas famílias antes do bloqueio, dificilmente podem ajudar seus pais neste momento de crise (ISLAM et al., 2020; VAN HARMELEN et al., 2016).

Os achados sobre a triagem positiva de ansiedade foram maiores do que em estudo realizado por Chirikov et al., (2020) (GAD-2 $\geq$ 3) em estudantes universitários durante a pandemia da COVID-19 na Califórnia que foi de $35 \%$, enquanto no mesmo estudo, $39 \%$ dos estudantes apresentaram depressão provável (PHQ-2 $\geq 3$ ) próximo ao encontrado em nosso estudo (CHIRIKOV et al., 2020).

Enquanto em um estudo realizado na Itália por Gualano et al., (2020) durante a COVID-19 foi identificado que $23,6 \%$ e $24,7 \%$ dos universitários tiveram triagem positiva de ansiedade (GAD-2 $\geq 3$ ) e depressão (PHQ-2 $\geq$ 3), respectivamente; estes resultados se encontram mais baixos dos que os encontrados na presente pesquisa em ambos os transtornos (GUALANO et al., 2020).

Assim, esses achados revelam taxas significativas de estudantes com triagem positiva de ansiedade e depressão, e que relataram sentir sintomas de ansiedade e depressão quase todos os dias nas últimas duas semanas, isto pode estar relacionado ao efeito causado pelo isolamento social, o qual, ocasiona sentimentos de solidão, de vulnerabilidade e preocupação, levando a se sentirem menos eficientes na superação do problema, bem como, fatores como: informações transmitidas pelos diversos meios (número de óbitos e de casos positivos de COVID-19), mesmo se sabendo que estes não fazem parte dos grupos com maior risco em termos de letalidade (MAIA; DIAS, 2020; MARELLI et al., 2020; WEISS; MURDOCH, 2020; ZHOU et al., 2020).

O fechamento das universidades pode ter contribuído para essas taxas, já que causou atrasos acadêmicos, levando a maior probabilidade de se formarem mais tarde do que o esperado (ISLAM et al., 2020). Além disso, a mudança no método de ensino para o ensino online gerou cargas de estudo estressantes que podem gerar sintomas de ansiedade e depressão, concomitantemente com fatores como: muitas atribuições e requisitos a cumprir, falta de recursos 
financeiros para uma internet de qualidade e a queda de internet e/ou energia, podem ter contribuído para gerar ainda mais esses sintomas (FAWAZ; SAMAHA, 2021; KASSE; KASSE; BALUNYWA, 2013).

As aulas online foi uma alternativa de ensino viável em tempos de quarentena, onde os alunos ainda poderiam se beneficiar ao atualizar seus cursos, no entanto, na área de ciência da saúde certos tópicos são muito mais difíceis de entregar de forma online, principalmente aqueles que envolvem aspectos práticos ou mesmo clínicos, assim o fechamento de universidades veio para manter os estudantes seguros, no entanto, essas noções surgiram com diferentes conjuntos de problemas de saúde mental (CHIRIKOV et al., 2020; FAWAZ; SAMAHA, 2021; ISLAM et al., 2020).

A prevalência de uso de psicofármacos entre os estudantes foi menor que 0 encontrado em um estudo realizado por Boclin et al., (2020) que foi de $24,7 \%$, isso pode ter sido pela menor quantidade de entrevistados no presente estudo. Para os jovens, a universidade é um passo importante em uma sociedade cada vez mais competitiva e desafiadora (BOCLIN et al., 2020). Durante o curso acadêmico, os alunos sentem-se pressionados para ter sucesso e precisam se superar continuamente, assim, a melhora da concentração, da memória e da atenção é a principal necessidade que leva os universitários ao uso de psicofármacos (BOCLIN et al., 2020).

Houve maior frequência de uso de antidepressivos, sendo os inibidores da recaptação da serotonina os mais utilizados. Estes populares antidepressivos são geralmente usados para o tratamento de depressão ou ansiedade (MEJÍA; RESTREPO; BERNAL, 2018). Enquanto a maior prevalência de uso de psicofármacos por motivos associados, pode indicar o uso indevido de medicamentos psicofármacos, que deve ser avaliado por profissional médico para adequação da farmacoterapia (BOCLIN et al., 2020).

No que diz respeito ao tempo de utilização dos psicofármacos, a grande maioria relatou a utilização deles, a mais de 1 ano, entre eles os benzodiazepínicos, cabe ressaltar que o uso dessa classe de medicamentos de forma crônica pode causar efeitos colaterais como tolerância, dependência e crises de abstinência. Assim, é recomendado que sejam utilizados por um curto período, cerca de 4 a 6 semanas (MOSFIAK; BRZOZOWSKI; CICHOTA, 2020).

Houve uma quantidade significativa de universitários que não associam o uso de psicofármacos com a COVID-19, sendo assim outros fatores podem ter levado a esse uso. Vale destacar que houve uma quantidade significativa de estudantes que não faziam uso de psicofármacos e que foram classificados com provável ansiedade e depressão, assim indica que muitos não estão tratando da doença ou buscam outras formas de tratamento.

\section{CONCLUSÃO}

Os resultados deste estudo sugerem que os alunos de graduação estão enfrentando desafios de saúde mental significativos durante a pandemia COVID 19. Evidenciando, que boa parte dos estudantes foram avaliados com transtorno depressivo provável e/ou transtorno de ansiedade provável. Fatores como incerteza na vida acadêmica e profissional, carga estressante de ensino online e a insegurança financeira podem ter contribuído para esses resultados. Enquanto que foi identificada uma pequena porcentagem de estudantes fazendo uso de psicofármacos, podendo sugerir outras formas de tratamentos ou a falta de tratamento.

Assim diante desse cenário cabe ao governo e a administração das universidades trabalharem juntos para fornecer apoio psicológico aos estudantes universitários. É importante também o apoio dos pais que devem ser incentivadores e devem criar um vínculo familiar amigável e positivo para seus filhos, sem impor pressão sobre a futura carreira acadêmica e profissional.

Apesar da limitação de ter utilizado um instrumento de triagem, este estudo possibilitou $\mathrm{o}$ rastreamento de ansiedade $\mathrm{e}$ depressão entre estudantes universitários 
durante a pandemia causada pela COVID-19. Outra limitação deste estudo foi a amostragem não probabilística, pois foi escolhida a amostragem por conveniência, devido à baixa taxa de resposta e a sensibilidade ao tempo do surto COVID-19. Contudo, é sugerido que os alunos com uma pontuação de GAD-2 e PHQ2 , maior ou igual a 3 , procure apoio psicológico para avaliações adicionais.

\section{AGRADECIMENTOS}

Aos estudantes, professores e servidores da Universidade Federal de Sergipe, Lagarto-SE.

\section{CONFLITOS DE INTERESSES}

Os autores declaram que a presente pesquisa foi realizada na ausência de quaisquer conflitos de interesses.

\section{REFERÊNCIAS}

ALSHARJI, K. E. Anxiety and depression during the COVID-19 pandemic in Kuwait: the importance of physical activity. Middle East Current Psychiatry, v. 27, n. 60, 2020. DOI: 10.1186/s43045-020-00065-6.

BASHIR, T. F. et al. The psychological impact analysis of novel COVID-19 pandemic in health sciences students: A Global Survey. European Journal of Dentistry, v. 14, 2020. DOI: 10.1055/s-0040-1721653.

BOCLIN, K. L. S. et al. Academic performance and use of psychoactive drugs among healthcare students at a university in southern Brazil: cross-sectional study. São Paulo: Medical Journal, v. 138, n. 1, p. 27-32, 2020. DOI: 10.1590/15163180.2019.0182.R1.21102019.

BRASIL, Conselho Nacional de Saúde. Resolução no 510, de 07 de Abril de 2016 Dispõe sobre a pesquisa em Ciências Humanas e Sociais, 7 abr. 2016.

BRASIL, Ministério da Saúde. Primeiro caso de Covid-19 no Brasil permanece sendo o de 26 de fevereiro. 2020. Disponível em: https://www.saude.gov.br/noticias/agenciasaude/47215-primeiro-caso-de-covid-19-nobrasil-permanece-sendo-o-de-26-de-fevereiro. Acesso em: 15 ago. 2020.

CHIRIKOV, I. et al. Undergraduate and graduate students' mental health during the COVID-19 pandemic. EScholarship: UC Berkeley. Disponível em: https:// escholarship.org/uc/item/80k5d5hw. Acesso em: 14 jan. 2021.

FAWAZ, M.; SAMAHA, A.. E-learning: depression, anxiety, and stress symptomatology among Lebanese university students during COVID-19 quarantine. Nursing Forum, v. 56, n. 1, p. 52-57, 2021. DOI: 10.1111/nuf.12521.

GRUBIC, N.; BADOVINAC, S.; JOHRI, A. $M$. Student mental health in the midst of the COVID-19 pandemic: A call for further research and immediate solutions. International Journal of Social Psychiatry, v. 66, n. 5, p. 517-518. DOI: $10.1177 / 0020764020925108$.

GUALANO, M. R. et al. Effects of COVID19 lockdown on mental health and sleep disturbances in Italy. International Journal of Environmental Research and Public Health, v. 17, n. 13 , p. 4779 , 2020. DOI: $10.3390 /$ ijerph17134779.

ISLAM, M. A. et al. Depression and anxiety among university students during the COVID-19 pandemic in Bangladesh: A webbased cross-sectional survey. PLOS ONE, v. 15, n. 8, p. e0238162, 2020. DOI: 10.1371/ journal.pone. 0238162 .

KASSE, J.; BALUNYWA, W. An assessment of e-learning utilization by a section of Ugandan universities: challenges, success factors and way forward. International conference on ICT for Africa, 2013.

KROENKE, K.; SPITZER, R. L.; WILLIAMS, J. B. The patient health questionnaire-2: Validity of a two-item 
depression screener. Medical Care, v. 41, n. 11 , p. 1284-1292, 2003. DOI: 10.1097/01.MLR.0000093487.78664.3C.

KROENKE, K. et al. Anxiety disorders in primary care: Prevalence, impairment, comorbidity, and detection. Annals of Internal Medicine, v. 146, n. 5, p. 317-325, 2007. DOI: 10.7326/0003-4819-146-5-200703060-00004.

LELIS, K. C. G. et al. Sintomas de depressão, ansiedade e uso de medicamentos em universitários. Revista Portuguesa de Enfermagem de Saúde Mental, n. 23, p. 914, 2020. DOI: 10.19131/RPESM.0267.

LIU, X. et al. Psychological status and behavior changes of the public during the COVID-19 epidemic in China. Infectious Diseases of Poverty, v. 9, n. 1, p. 58, 2020. DOI: 10.1186/s40249-020-00678-3.

MAIA, B. R. DIAS, P. C. Anxiety, depression and stress in university students: The impact of COVID-19. Estudos de Psicologia (Campinas), v. 37, 2020. DOI: 10.1590/1982-0275202037e200067.

MARELLI, S. Impact of COVID-19 lockdown on sleep quality in university students and administration staff. Journal of Neurology, v. 268 , n. 1 , p. $8-15,2020$. DOI: 10.1007/s00415-020-10056-6.

MEJÍA, C. B. M.; RESTREPO, M. L.; BERNAL, D. R. Actitudes, conocimientos y prácticas frente a la automedicación con productos herbales y psicofármacos en estudiantes de medicina de Medellín-Colombia. MEDICINA U.P.B., v. 37, n. 1, p. 17-24, 2018. DOI: 10.18566/medupb.v37n1.a03.

MENDONÇA, G. A. Níveis de depressão, ansiedade e estresse em estudantes de enfermagem e intervenções para manejo. 2020. 79 f. Dissertação (Mestrado) - Escola de Enfermagem de Ribeirão Preto, Universidade de São Paulo, Ribeirão Preto, 2020.
MOSFIAK, M. A. et al. Analysis of benzodiazepine consumption in a city of Northern Rio Grande. Revista de Saúde Coletiva da UEFS do Rio Grande do Sul, Brasil, v. 10, p. 49-57, 2020. DOI: 10.13102/ rscdauefs.v10.5214.

MOUSTAFA, A. A. et al. Impulsivity and its relationship with anxiety, depression and stress. Comprehensive Psychiatry, v. 74, p. 173-179, 2017. DOI: 10.1016/ j.comppsych.2017.01.013.

$\mathrm{NI}, \mathrm{M}$. Y. et al . Mental health, risk factors, and social media use during the COVID-19 epidemic and cordon sanitaire among the community and health professionals in Wuhan, China: cross-sectional survey. JMIR Mental Health, v. 7 , n. 5, p. e19009, 2020. DOI: 10.2196/19009.

OPAS/OMS. OPAS/OMS Brasil - Folha informativa - COVID-19 (doença causada pelo novo coronavírus). 2020. Disponível em: https://www.paho.org/bra/index.php? option=com_content\&view=article\&id=6101:cov id19\&ltemid=875. Acesso em: 8 set. 2021.

PEREIRA, M. D., et al. A pandemia de COVID-19, o isolamento social, consequências na saúde mental e estratégias de enfrentamento: uma revisão integrativa. Research, Society and Development, v. 9, n. 7, p. e652974548, 2020. DOI: 10.33448/rsdv9i7.4548.

ROLLEMBERG, G. S. M. Avaliação da presença de sintomas de ansiedade e depressão em estudantes de medicina da Universidade Federal de Sergipe, Campus Lagarto. 2018. Trabalho de conclusão de curso (Bacharelado), Departamento de Medicina - Universidade Federal de Sergipe, Campus Professor Antônio Garcia Filho, Lagarto/SE, 2018.

SANTIAGO, M. B. Índices de depressão, ansiedade e estresse entre estudantes de enfermagem e medicina do Acre. Revista Psicologia, Diversidade e Saúde, v. 10, n. 1, 
p. 73-84, 2021. DOI: 10.17267/23173394RPDS.V10I1.3374.

SANTINI, Z. I. et al. Social disconnectedness, perceived isolation, and symptoms of depression and anxiety among older Americans (NSHAP): a longitudinal mediation analysis. Articles Lancet Public Health, v. 5 n. 1, p. $62-70,2020$. DOI: 10.1016/ S2468-2667(19)30230-0.

SES, Secretaria do Estado de Saúde de Sergipe. Governo de Sergipe confirma primeiro caso de coronavírus. 2020. Disponível em: https://www.saude.se.gov.br/? p=39206. Acesso em: 15 ago. 2020.

SES, Secretaria do Estado de Saúde de Sergipe. Sergipe registra 149 casos de Covid-19 e mais quatro óbitos que estavam em investigação. 2021. Disponível em: https:// www.se.gov.br/noticias/saude/

sergipe_registra_149_casos_de_covid_19_e_ mais_quatro_obitos_que_estavam_em_investi gacao. Acesso em: 8 set. 2021.

SCHNEIDER, A. P. H.; AZAMBUJA, P. G. Uso de fármacos psicotrópicos por profissionais da saúde atuantes da área hospitalar. Infarma - Ciências Farmacêuticas, v. 27, n. 1, p. 14-21, 2015. DOI: 10.14450/2318-9312.v27.e1.a2015.pp14-21.

SMOLEN, J. R.; ARAÚJO, E. M. Raça/cor da pele e transtornos mentais no Brasil: uma revisão sistemática. Ciência \& Saúde Coletiva, v. 22, n. 12, p. 4021-4030, 2017. DOI: 10.1590/1413-812320172212.19782016.

SOUZA, D. C. Condições emocionais de estudantes universitários: estresse, depressão, ansiedade, solidão e suporte social. 2017. Dissertação (Mestrado em Psicologia) - Universidade Federal do Triângulo Mineiro, Uberaba/MG, 2017. Disponível em: http://bdtd.uftm.edu.br/bitstream/tede/507/5/ Dissert\%20Deise\%20C\%20Souza.pdf . Acesso em: 16 ago. 2020.

STONE, A. M.; MERLO, L. J. Attitudes of college students toward mental illness stigma and the misuse of psychiatric medications. Journal of Clinical Psychiatry, v. 72, n. 2, p. 134-139, 2011. DOI: 10.4088/ JCP.09m05254ecr.

STONE, A. M.; MERLO, L. J. Psychiatric medication-seeking beliefs and behaviors among college students. The American journal of drug and alcohol abuse, v. $38, n$. 4 p. $\quad 314-21, \quad 2012 . \quad$ DOI: 10.3109/00952990.2011.643992.

VAN HARMELEN, A. L. et al. Friendships and family support reduce subsequent depressive symptoms in at-risk adolescents. PLOS ONE, v. 11, n. 5, p. e0153715, 2016. DOI: 10.1371/journal.pone.0153715.

WEISS, P.; MURDOCH, D. R. Clinical course and mortality risk of severe COVID-19. The Lancet, 2020. DOI: 10.1016/S01406736(20)30633-4.

ZHOU, F. et al. Clinical course and risk factors for mortality of adult inpatients with COVID-19 in Wuhan, China: a retrospective cohort study. The Lancet, v. 395, n. 10229, p. 1054-1062, 2020. DOI: 10.1016/S01406736(20)30566-3. 